\title{
Korean Medical Professionals' Attitudes and Experiences on Advance Care Planning for Noncancerous Disease
}

\author{
Ji Eun Lee ${ }^{1}$, Aejin Goo $^{2}$, Dong Wook Shin ${ }^{3,4}$, Jun Hyun Yoo ${ }^{3}$; Korean Geriatrics Society End-of-Life Research Group \\ ${ }^{1}$ Department of Family Medicine, CHA Bundang Medical Center, CHA University, Seongnam, Korea \\ ${ }^{2}$ Department of Family Medicine, National Center for Mental Health, Seoul, Korea \\ ${ }^{3}$ Department of Family Medicine/Supportive Care Center, Samsung Medical Center, Sungkyunkwan University School of Medicine, Seoul, Korea \\ ${ }^{4}$ Department of Digital Health, Samsung Advanced Institute for Health Sciences \& Technology, Sungkyunkwan University, Seoul, Korea
}

Corresponding Author:

Dong Wook Shin, MD, DrPH, MBA

https://orcid.org/0000-0001-8128-8920

Department of Family Medicine/

Supportive Care Center, Samsung

Medical Center, Sungkyunkwan

University School of Medicine,

Department of Digital Health, SAIHST,

Sungkyunkwan University, 81 Irwon-

ro, Gangnam-gu, Seoul 06351, Korea

E-mail: dwshin.md@gmail.com

Co-corresponding Author:

Jun Hyun Yoo, MD, MPH, PhD

https://orcid.org/0000-0002-0344-5123

Department of Family Medicine/

Supportive Care Center, Samsung

Medical Center, Sungkyunkwan

University School of Medicine, 81

Irwon-ro, Gangnam-gu, Seoul 06351,

Korea

E-mail: drjohn.yoo@samsung.com

Received: March 18, 2019

Revised: April 19, 2019

Accepted: May 22, 2019
Background: Advance care planning (ACP) allows patients to declare their preferences for life-sustaining and hospice palliative care. However, the perception of ACP remains low in Korea. The present study assessed the attitudes and status of medical professionals in relation to end-of-life care decisions in older and noncancerous patients. Methods: This descriptive correlation study was performed to understand the attitudes regarding and status of ACP and advance directives (AD). For this purpose, we conducted a survey of members who attended the Spring Conference of the Korean Geriatrics Society in May 2015 using a questionnaire that included questions on experiences related to AD, opinions on disturbance factors and improvement measures, and questions about the status of their medical institutions. Results: All of 181 respondents were doctors. Among the respondents, $21.7 \%$ had the experience of treating patients who had completed an AD. Medical professionals saw AD use as appropriate for terminal patients with less than 6 months of life expectancy, as well as those with degenerative neurological disorders such as amyotrophic lateral sclerosis, chronic diseases such as chronic renal disease, and early stages of Alzheimer's disease. Conclusion: The results showed that geriatrics medical professionals agreed with the necessity for $A D$ in noncancerous terminal diseases and that consideration of a family-centered decision-making culture, legal protection for medical professionals, and education of the general public and medical professionals will be helpful for the popularization of AD. (Ann Geriatr Med Res 2019;23:63-70)

Key Words: Advance directives, Aged, Attitude of health personnel, Life-sustaining medicine, Noncancerous disease

\section{INTRODUCTION}

Since the 1997 "Boramae Case" in Korea that applied accessory to murder to medical professionals who stopped life-sustaining treatment ${ }^{1)}$ and the "Grandma Kim Case" in 2009 that first recognized the termination of lifesustaining treatment, ${ }^{2)}$ social discussion about the selfdetermination rights of patients regarding terminal-stage medical care has increased. Therefore, the need to complete physician orders for life-sustaining treatment (POLST) or advance directives (AD) to guarantee patients' self-determination has been suggested. ${ }^{3)}$ As a result, the "Hospice, Palliative Care, and Life-Sustaining Treatment DecisionMaking Act" (hereafter referred to as "Life-Sustaining Treatment Decision-Making Act") was established in February 2016. This policy was first enacted in August 2017 and was adopted in all medical institutions on February 4, 2018.

Life-sustaining treatment refers to cardiopulmonary resuscitation, blood dialysis, anti-cancer drug administration, and use of ventilators in patients who are unlikely to recover. This can be seen as extending the end-of-life process through medical procedures such as equipment or medications without treatment effects. The "Life-Sustaining Treatment Decision-Making Act" enables patients to indicate their decision to defer or terminate life-sustaining treatment through POLST. Upon the determination of terminal stage or end-of-life status, the physician (doctor) prepares a POLST at the request of the patient. This is a sign that life-sustaining treatment will not be performed. POLST takes over when a patient cannot express his or her opinion due to conditions such as unconsciousness. 
$\mathrm{AD}$ refers to a document that the patient defines a priori after receiving sufficient medical information from the medical staff when the patient has decision-making ability about the medical action they will receive. ${ }^{4)}$ This document generally comprises treatment and proxy directives. The treatment directive describes the types of treatment desired by the patient, while the proxy directive is the designation of the individual who will make decisions once the patient loses decision-making abilities. Through AD, the patient can indicate their preferences about and document lifesustaining treatments and hospice palliative care.

While discussions about terminal-stage medical care have generally been limited to terminal-stage cancer patients, the "Life-Sustaining Treatment Decision-Making Act" has expanded their scope to include non-cancer terminal-stage patients including those with AIDS, chronic obstructive pulmonary disease, and liver cirrhosis. As the cause of death from geriatric diseases increases with aging population, both cancer and noncancerous chronic diseases are important targets of palliative care. Due to the long disease duration of noncancerous chronic illnesses in older individuals, ${ }^{5)}$ a palliative care approach, rather than medical service such as hospitalization as in the case of cancer, is important. For this, it is necessary to deal with $\mathrm{AD}$ from the beginning of treatment. ${ }^{6}$ In particular, problems with unclear prognosis, cognitive function, and various clinical situations in older people require different approaches from those applied in terminal cancer patients.

The present study investigated medical professionals specializing in geriatric illnesses to examine their perceptions of terminal-stage medical care and to explore their opinions about the popularization of $\mathrm{AD}$. As previous studies have been limited to terminal-stage cancer and there remains insufficient research on older adults with chronic diseases other than terminal-stage cancer, we focused on non-cancer chronic diseases other than terminalstage cancer.

\section{MATERIALS AND METHODS}

The present descriptive correlation study examined the attitudes and the status of Advance Care Planning (ACP) among medical professionals.

\section{Research Participants}

The study was conducted among medical professionals attending the 55th Spring Conference of the Korean Geriatrics Society. Those who understood the purpose of the study and agreed to participate were selected and there were no particular exclusion criteria. Because the status was assessed descriptively with a cross-sectional analysis for the investigation of perception, a specific sample size calculation was not necessary. The study was approved by the institutional review board (IRB) at Seoul National University Hospital (IRB No. 1504-107-666).

\section{Data Collection Method}

To collect data for this study, we explained the purpose of research, obtained consent, and distributed surveys in two sessions assigned across two days of the conference on May 30 and 31, 2015. The questionnaires were selfreported and a researcher provided help if necessary.

\section{Research Tool}

This study developed and applied a questionnaire to investigate the perception of medical professionals. ${ }^{7-10)}$ The questionnaire contained a total of 31 items that could be broadly categorized into $\mathrm{AD}$-related experiences, opinions about hindering factors and opportunities for improvement, questions about the current status of the affiliated medical institution, and questions related to the respondent. For assessment of AD-related experiences, we asked whether the participants had experienced treating patients who had completed an $\mathrm{AD}$, how much the $\mathrm{AD}$ was followed if it was completed, the reasons for not following the $\mathrm{AD}$, and whether the treatment plan had been changed due to the $\mathrm{AD}$; whether the timing or frequency of conversations with patients or caretakers increased after confirming the $\mathrm{AD}$, what proportion of $\mathrm{AD}$ discussions occurred when treating terminal-stage patients, who brought up the topic of discussion, and the reasons that the discussion was not brought up; and whether the participant had experienced receiving a Do Not Resuscitate (DNR) from the caretaker and the reasons for the DNR. For opinions about $\mathrm{AD}$, we asked whether the participants agreed with the popularization of $\mathrm{AD}$, the reason for their agreement or disagreement, in which cases the $\mathrm{AD}$ should be considered, whether the participant believed that the $\mathrm{AD}$ discussion was the role of a medical professional, what is important for a respectable death, and the ideal location for end-oflife and the reasons for this belief. Regarding the current status of their affiliated medical institutions, we asked participants whether terminal patient care guidelines were in place at their institution, when they had been or were planned to be introduced, and the reasons for no plan for their introduction; whether there was an $\mathrm{AD}$ guideline in the institution, when it was introduced, when it was used, who it was used for, and whether there was plan for its introduction if it was not yet in use; whether job training had been provided for terminal patient care; and what methods existed for activation. Among questions related to the respondent, we asked whether they had completed their own $\mathrm{AD}$ or whether someone in their family had completed it; if the respondent had experience with $\mathrm{AD}$ training; the respondent's gender, birth year, and religion; and the type of affiliated medical institution.

\section{Data Analysis}

From the collected data, the general characteristics of the respondents, their frequencies of following $\mathrm{AD}$, the level of agreement according to disease, the level of agreement 
to terminate palliative care according to the situation, the reasons for not discussing $\mathrm{AD}$, the reasons for agreeing or disagreeing with the $\mathrm{AD}$, and the methods for $\mathrm{AD}$ popularization were determined by analyzing the frequencies and proportions (percentages). STATA version 14.1 (STATA Corp., College Station, TX, USA) was used to perform the analyses.

\section{RESULTS}

\section{General Participants Characteristics}

The general characteristics of participants are listed in Table 1 . The average age was 46.9 years and $70.2 \%$ were men. All 181 respondents indicated that they were doctors. The types of affiliated institutions were convalescent hospitals (78, 43.1\%), university hospitals (52, 28.7\%), general hospitals excluding university hospitals (21, 11.6\%), primary clinics $(20,11.1 \%)$, and public health centers and others $(10,5.5 \%)$.

\section{Experiences with AD}

Most of the respondents lacked experience with $\mathrm{AD} ; 7.2 \%$ responded that they had completed an $\mathrm{AD}$, while 6.7\% responded that someone in their family had completed it. Only $16.6 \%$ had experienced AD-related training in their medical education.

Of the $21.7 \%$ of respondents with experience treating patients who had completed an $\mathrm{AD}$, the majority (66.7\%) followed most of the $\mathrm{AD}$ content in patient treatment decision-making (Fig. 1). The reasons for not following the $\mathrm{AD}$ included family opposition (38.5\%), AD content that did not fit the medical situation at that time (35.9\%), and the possibility of disease recovery (30.8\%). The majority (62.5\%) of those who responded that they had experienced treating patients who had completed an AD reported

Table 1. Participant characteristics $(n=181)$

\begin{tabular}{|lc|}
\hline \multicolumn{1}{|c|}{ Variable } & Value \\
\hline Age (y) & $46.9 \pm 12.4$ \\
\hline Gender & $127(70.2)$ \\
\hline Men & $54(29.8)$ \\
\hline Women & \\
\hline Religion & $48(26.5)$ \\
\hline Protestantism & $45(24.9)$ \\
\hline Catholicism & $19(10.5)$ \\
\hline Buddhism & $1(0.6)$ \\
\hline Others & $68(37.6)$ \\
\hline None & $20(11.1)$ \\
\hline Affiliation & $78(43.1)$ \\
\hline Primary clinic & $52(28.7)$ \\
\hline Convalescent hospital & $21(11.6)$ \\
\hline University hospital & $10(5.5)$ \\
\hline General hospital & \\
\hline Public health center, others & \\
\hline
\end{tabular}

Values are presented as mean \pm standard deviation or number (\%). Total percentages may not equal 100\% due to rounding. changing the treatment plan due to the $\mathrm{AD}$. An increased frequency of conversations with patients and caretakers was reported by $56.3 \%$ of respondents with experience with $\mathrm{AD}$.

In response to the question asking whether participants discussed $\mathrm{AD}$ when treating terminal patients, 31.3\% reported that they did not, $36.9 \%$ responded that they did so less in than 50\% of cases, and 31.9\% responded that they discussed $\mathrm{AD}$ in at least 50\% of cases. The doctors most commonly initiated the discussion (44.2\%), followed by caretakers (29.3\%) and patients (13.8\%). The reasons for not initiating discussions about AD included, "I'm afraid if I do not discuss it with my family, I will have a legal problem later" (60.2\%), "The patient may feel depressed by feeling hopeless" (57.5\%), "The patient will interpret this discussion as giving up on treatment" (55.8\%), and "I believe that the caretaker has the right to determine the treatment direction of the patient" (50.3\%) (Table 2).

Among participants, 85.1\% reported that they receive a DNR from caretakers, rather than from patients themselves. The reasons included a lack of patient decisionmaking ability (76.2\%), worry about future legal problems (49.7\%), consideration of the patient's psychological pain $(45.8 \%)$, respecting the role of the caretaker in making treatment decisions (35.9\%), the possibility that patient will view it as giving up on treatment (30.9\%), emotional pressure about asking the patient (23.8\%), family opposition about receiving it from the patient (21.5\%), and insufficient rapport with the patient (13.3\%).

\section{Opinions about AD}

When asked if they agreed or disagreed with $\mathrm{AD}$ considering the environment in Korea, 87.2\% of the participants agreed and 10.6\% disagreed. The reasons for agreement (multiple selection), included "It is important to have patients who may lose their ability to make decisions to determine their treatment plan" (49.7\% of all respondents),

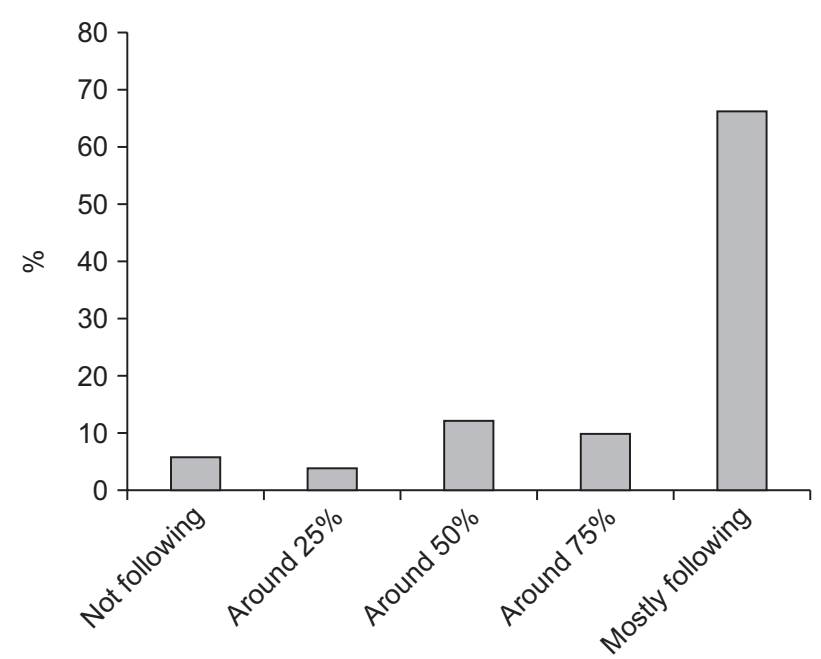

Fig. 1. Frequency of following advance directives when it is prepared. 
Table 2. Reasons for not initiating discussions about advance directives (multiple responses)

\begin{tabular}{|l|c|}
\hline \multicolumn{1}{|c}{ Reasons } & Number (\%) \\
\hline a. Patient factors & 104 (57.5) \\
\hline The patient may feel depressed by feeling hopeless & 101 (55.8) \\
\hline The patient will interpret this discussion as giving up on treatment & 81 (44.8) \\
\hline The patient will be anxious if he or she discusses advance directives & 66 (36.5) \\
\hline The patient was unable to make a decision & 53 (29.3) \\
\hline My patients are not fully prepared & 41 (22.7) \\
\hline The patient will get angry & $30(16.6)$ \\
\hline The patient may choose to die if we discuss death & 109 (60.2) \\
\hline b. Family factors & 91 (50.3) \\
\hline I'm afraid if I do not discuss it with my family, I will have a legal problem later & 70 (38.7) \\
\hline I believe that the caretaker has a right to determine the treatment direction of the patient & 50 (27.6) \\
\hline Family members will object to this discussion & $79(43.6)$ \\
\hline If I discuss it with a patient, my family will criticize me for the patient's decision & 74 (40.9) \\
\hline c. Physician factors & 57 (31.5) \\
\hline If you stop the patient's life-sustaining treatment, it could cause a legal problem & 50 (27.6) \\
\hline It is difficult to predict and explain the outcome & 49 (27.1) \\
\hline Rapport is not formed with patients to discuss advance directives & 41 (22.7) \\
\hline It is emotionally difficult to discuss & 31 (17.1) \\
\hline Discussion of advance directives is like defending euthanasia & \\
\hline I myself do not fully understand the meaning of advanced directives & \\
\hline We have no time to discuss it & \\
\hline
\end{tabular}

Table 3. Opinions on advance directives (multiple responses)

\begin{tabular}{|lc|}
\hline \multicolumn{1}{|c}{ Opinions } & Number (\%) \\
\hline a. Reasons for approval of advance directives & $90(49.7)$ \\
It is important to have patients who may lose their ability to make & $35(19.3)$ \\
decisions to determine their treatment plan in advance & $25(13.8)$ \\
\hline It provides help in caring for patients at their terminal stage & $18(9.9)$ \\
\hline It helps patients feel that they can control their lives & $1(0.6)$ \\
\hline It helps lessen the pressure of caretakers regarding decision-making & 18 \\
\hline Others & $15(38.5)$ \\
\hline No response & $14(35.9)$ \\
b. Reasons for opposition to advance directives & $12(30.8)$ \\
\hline It makes the patient lose hope & $3(7.7)$ \\
\hline It is akin to supporting euthanasia & $2(5.1)$ \\
\hline There can be legal problems of terminating palliative care & 142 \\
\hline Terminating palliative care can represent giving up on the patient & \\
\hline Others & \\
\hline No response & \\
\hline
\end{tabular}

followed by "It provides help in caring for patients at their terminal stage" (19.3\%), "It helps patients feel that they can control their lives" (13.8\%), and "It helps lessen the pressure of caretakers regarding decision-making” (9.9\%). The reasons for disagreeing with $\mathrm{AD}$ (multiple selections possible) included "It makes the patient lose hope" (38.5\% of respondents), "It is akin to supporting euthanasia" (35.9\%), "There can be legal problems of terminating palliative care" (30.8\%); and "Terminating palliative care can represent giving up on the patient" (7.7\%) (Table 3).

When asked for opinions on which cases should be considered for $\mathrm{AD}$ completion (multiple selections), most agreed with completion for terminal patients with less than 6 months of life expectancy (96.7\%) and the majority agreed with $\mathrm{AD}$ completion for degenerative neurological disorders such as amyotrophic lateral sclerosis (69.0\%), chronic diseases such as chronic renal disease (56.6\%), and early stages of Alzheimer's (53.0\%) (Fig. 2).

Most participants (81.7\%) felt that the AD discussion was the role of the medical professional, while the rest (18.3\%) responded that they are not sure.

Regarding life-sustaining treatment, $67.4 \%$ and 70.7\% of participants had negative opinions about doctor-assisted suicide and active euthanasia, respectively. In contrast, $27.1 \%$ felt that it was ethically feasible to terminate palliative care for terminal-stage patients with decision-making 


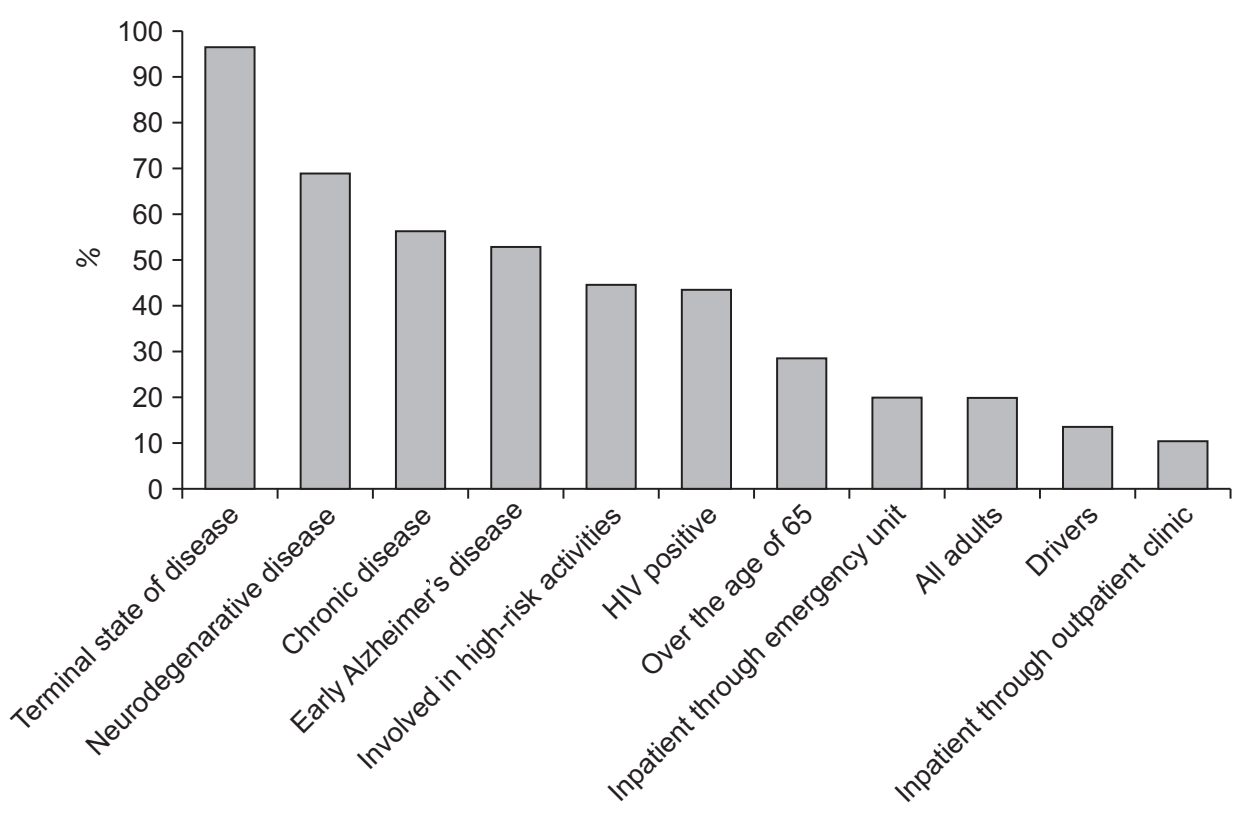

Fig. 2. Agreement of advance directives for each disease and situation.

Table 4. Strategies for the activation of advance directives

\begin{tabular}{|c|c|c|c|c|}
\hline Strategies & Will be helpful & Will not be helpful & Will interfere & No response \\
\hline Establishment of legal protection & $172(95.6)$ & $8(4.4)$ & $0(0)$ & 1 \\
\hline Education of medical professionals & $169(93.9)$ & $11(6.1)$ & $0(0)$ & 1 \\
\hline Education of the general public & $164(91.1)$ & $15(8.3)$ & $1(0.56)$ & 1 \\
\hline Legalization of advanced directive completion & $143(82.2)$ & $25(14.4)$ & $6(3.5)$ & 7 \\
\hline Policy support for the preparation of advance directives in hospital & $141(81)$ & $30(17.2)$ & $3(1.7)$ & 7 \\
\hline Rewards for time spent discussing advance directives & $130(74.7)$ & $40(23)$ & 4 (2.3) & 7 \\
\hline $\begin{array}{l}\text { Hospital policy ready for all patients to } \\
\text { complete advance directives on admission }\end{array}$ & 98 (57) & $60(34.9)$ & $14(8.1)$ & 9 \\
\hline
\end{tabular}

Values are presented as number (\%).

ability and 49.2\% felt that it could be justified depending on the situation, showing flexibility in a large number. However, 35.9\% felt that terminating the life of a terminal patient who does not have decision-making ability and did not make the request absolutely cannot be justified, while $41.4 \%$ felt that it could be justified depending on the situation.

Investigation of the perceptions about the factors related a respectable death revealed that "Being with family or significant persons" (97.3\% of participants), "Surroundings have been wrapped up" (95.6\%), "Being free of pain" (92.8\%), "Having lived a significant life" (83.0\%), "Not pressuring others" (82.3\%), "Spiritual relaxation" (81.3\%), "Choosing my own treatment method" (72.0\%), "Obtaining sufficient finances" (61.5\%), "Clear consciousness until the end" (42.9\%), and "Dying at home" (30.2\%) were most important.

The ideal locations of death were hospice institution (37.5\%), hospital (30.7\%), home (28.4\%), and nursing facilities (2.8\%). The ideal location provided a peaceful death and appropriate care, according to $94.0 \%$ of respondents who chose hospice institution, $79.6 \%$ of respondents who chose hospitals, and $80.0 \%$ of respondents who chose nursing facilities. Those who selected the home as the ideal location felt that it allowed death in a more personable setting (58.0\%) and because loving families could witness the death (38.0\%).

\section{Current Status of AD in Medical Institutions}

To determine the current status of $\mathrm{AD}$ in the affiliated medical institution, we asked participants whether there were terminal patient care guidelines at their institution, when they were introduced, whether there was a plan for introduction if they were not yet in place, and the reasons for no plan for their introduction; whether there were $A D$ guidelines in place at their institution, when they were introduced, when they were used, to what cases $\mathrm{AD}$ were applied, whether there was plan for their introduction if they were not yet in place; and whether job training was provided for the care of terminal patients.

A lack of terminal patient care guidelines was reported by $55.8 \%$ of respondents, while $21 \%$ reported that there were guidelines and 23.2\% responded that they did not know if there were guidelines. Among participants whose 
institutions lacked these guidelines, $28.2 \%$ indicated that there was a plan for their implementation, while $59.1 \%$ did not know if there was a plan to introduce these guidelines.

Regarding AD guidelines, 29.8\% of participants responded that $\mathrm{AD}$ guidelines existed at their affiliated institution, 49.2\% reported that guidelines did not exist, and 21.0\% responded that they did not know. Among the affiliated institutions with $\mathrm{AD}$ guidelines, 40.0\% reported using them at the terminal time point and $30.9 \%$ reported using them at the time of hospitalization. AD were received from caretakers in 52.7\% of cases, from patients in $18.2 \%$ of cases, and from both patients and the caretakers in $18.2 \%$ of cases.

When asked whether there was a plan to introduce $A D$ guidelines in the affiliated institutions without them, $20.4 \%$ responded that there was a plan, while $11.1 \%$ responded that there was not a plan and $68.5 \%$ responded that they did not know. When asked whether they were provided job training on terminal patient care in their institutions, 29.3\% responded that it was conducted, 28.7\% responded that it was not conducted, and 24\% responded that they did not know.

\section{Opinions about Methods of AD Popularization}

In response to the questionnaire item about what methods exist to popularize $\mathrm{AD}$, participants responded that the establishment of legal protection (95.6\%), education of medical professionals (93.9\%), education of the general public (91.1\%), and legalization of $\mathrm{AD}$ completion (82.2\%) would be helpful (Table 4).

\section{DISCUSSION}

To our knowledge, the present study is the first domestic study focusing on noncancerous chronic disease to investigate the perceptions of geriatric medical professionals regarding ACP. In particular, during the initial implementation of the "Life-Sustaining Treatment Decision-Making Act," examining the perceptions and hindrance factors of $\mathrm{AD}$ use in noncancerous diseases will be important to address such problems and enable the smooth enactment of this act.

\section{Experiences with $A D$ and Hindering Factors}

Our results revealed that most medical professionals lacked experience with $\mathrm{AD}$. Although $\mathrm{AD}$ are completed and used in some hospitals, their use in real clinical settings was low. The majority (52.6\%) of doctors who participated in our study indicated that they rarely (less than 25\% of cases) discussed $\mathrm{AD}$ when treating terminal patients.

The use of $\mathrm{AD}$ in Asia is low compared to that in Western cultures. According to a cross-cultural survey study ${ }^{12)}$ of medical professionals and patients, the $\mathrm{AD}$ completion rates were 79\% and 9\% in the United States and Japan, respectively; when asked about the usefulness of $\mathrm{AD}$ in palliative care, 100\% of the respondents in the United States indicated that $\mathrm{AD}$ were useful, compared to $71 \%$ of the respondents in Japan. In one study on the attitudes of medical professionals regarding $\mathrm{AD},{ }^{13)}$ the opinions of families were identified as important in the Asian countries of Japan, Thailand, and Singapore. This finding may be due to the cultural background of Asia in which family-centered decision-making has a large impact on the establishment of patient treatment plans. In particular, families of patients are often critical of medical professionals if they discuss AD with the patient. ${ }^{9)}$ One study in Japan ${ }^{14)}$ reported that opposing the desires of the family constituents trying to extend the life of the patients and instead following the wishes of the patients represented in the $\mathrm{AD}$ was a difficult problem for medical professionals in a real clinical setting. In these cases, medical professionals are criticized by the family and become involved in legal lawsuits; thus, the doctors seek to protect themselves from conflict.

Family opposition was also the main reason for not closely adhering to $\mathrm{AD}$ in our study (38.5\%) and concern about legal problems with families was also the major reason for not initiating a discussion about $\mathrm{AD}$ (60.2\%). Therefore, as in other Asian countries, family-centered decision-making is important in ACP in Korea. Thus, the need to appropriately reflect this need in the popularization and institutionalization of $\mathrm{AD}$ has been confirmed.

\section{Opinions about AD}

Regarding $\mathrm{AD}$ use in patients with noncancerous terminal diseases, medical professionals saw $\mathrm{AD}$ use as appropriate for terminal patients with less than 6 months of life expectancy, degenerative neurological disorders such as amyotrophic lateral sclerosis, chronic diseases including chronic renal disease, and early stages of Alzheimer's disease. One-third of the respondents indicated the necessity for advanced completion of $\mathrm{AD}$ in people older than 65 years of age.

Our finding of the respondents' opinion on the necessity for $\mathrm{AD}$ completion in terminal patients was similar to results from other studies. In a study on $\mathrm{AD}$ experience and knowledge of 643 Canadians, ${ }^{7)}$ 96\% agreed with AD completion in the case of terminal patients with short life expectancy. Similarly, our results showed that $96.7 \%$ of respondents felt that $\mathrm{AD}$ were necessary. However, while 95\% of respondents in the Canadian study felt that AD completion was necessary for patients with chronic disease patients, only 56.6\% agreed in our study. Similarly, $85 \%$ of respondents in the Canadian study felt that $\mathrm{AD}$ were necessary for HIV-positive patients, compared to $43.9 \%$ of respondents in our study. As the Canadian study reflecting the medical situation in the 1990s, there may be some differences from the medical professionals of today in which life extension is possible due to improvements in technology for treating chronic diseases or infectious illnesses. However, only $28.9 \%$ of respondents in our study indicated that individuals over 65 years of age require $\mathrm{AD}$, compared to $77 \%$ of the respondents in the Canadian 
study.

In our study, medical professionals preferred hospice institutions and hospitals over homes as the ideal place of death given a relaxed death and appropriate treatment. In contrast, another study in the general public reported that the majority of the respondents preferred their homes as the ideal location for death, ${ }^{15)}$ highlighting the differences between medical professionals and the general public regarding the ideal place of death.

\section{Methods of AD Popularization}

When asked for their medical professional opinions about how to popularize $\mathrm{AD}$, participants responded that institutional supplementation such as legalization of $\mathrm{AD}$ completion and legal protection were necessary for institutionalization and popularization. An $\mathrm{AD}$ had been completed in about 70\% of older persons who died in 2010 after the legalization of $\mathrm{AD}$ in the United States. ${ }^{16)}$ The findings on popularization methods in the present study were similar to those of foreign research conducted in the $1990 \mathrm{~s}^{7)}$; however, compared to the education of the general public (92\%), education of medical professionals (90\%), establishment of legal protection (89\%), and legalization of completion (80\%) suggested by the foreign research, the primary method for popularization in the present study was the establishment of legal protection (95.6\%). Legal issues such as medical disputes are becoming increasingly more frequent and the burden on medical professionals is also increasing. The establishment of safe, legal, and institutionalized methods is necessary for the popularization of AD. In particular, policy supplementation and establishment are necessary to enact bills regarding life-sustaining treatment decision-making so that limitations such as agreement from all family members do not apply legal pressure to medical professionals.

Meanwhile, many respondents indicated that education for medical professionals and the general public would also help to popularize AD. Given that medical professionals have had little experience with $\mathrm{AD}$ and not many medical facilities have adopted this, this suggests that medical professionals recognize the need for such education and expect the popularization of $\mathrm{AD}$. Based on previous research results ${ }^{17)}$ that $\mathrm{AD}$ discussion increased from $3 \%$ to $17 \%$ in only 1.5 years after residency training, law institutionalization methods and $\mathrm{AD}$ education are important factors for the popularization of $\mathrm{AD}$.

This study has some limitations. First, this study was conducted of members of the Korean Geriatrics Society; thus, the research participants were limited. Second, this study was conducted via voluntary participation and the possibility that the attitudes and experiences of the respondents differed from those of non-respondents cannot be ignored. Third, there is a possibility of recall bias in the process of respondents recalling information from the past. Fourth, as most of the domestic medical professionals were not familiar with $\mathrm{AD}$ or prior medical plans, the participants of the study may have also had an insufficient understanding of the discussion relating to $\mathrm{AD}$.

Despite these limitations, this study is significant in that it was conducted to examine the perceptions of medical professionals regarding $\mathrm{AD}$ use in the context of noncancerous chronic disease. Given that the problems of unclear prognosis, cognitive function, and various clinical situations in older people require a different approach from that of terminal cancer patients, this approach is even more important. Further research including the analysis of factors related to AD use after the enactment of "LifeSustaining Treatment Decision-Making Act” is needed.

In Korea, "Hospice, Palliative Care, and Life-sustaining Treatment Decision-making Act," referred to as the "WellDying Act" was enacted in early 2016, and the policies of "Life-Sustaining Treatment Decision-Making Act" have been adopted in all medical institutions since February 4, 2018. ${ }^{18)}$ Legal conflicts between medical professionals and families about the termination of life-sustaining treatment and the medical costs for meaningless life-sustaining treatment are expected to decrease through this. Since the target population is not limited to patients with malignant diseases, we expect that the act will contribute to the popularization of hospice palliative care for noncancerous terminal diseases as well. This research showed that geriatrics medical professionals also agree with the necessity of $\mathrm{AD}$ in noncancerous terminal diseases and that consideration of family-centered decision-making culture, legal protection for medical professionals, and education for the general public and medical professionals will be helpful for the popularization of $\mathrm{AD}$.

\section{CONFLICTS OF INTEREST DISCLOSURES}

The researchers claim no conflicts of interest.

\section{ACKNOWLEDGMENTS}

This research was funded by Korean Geriatrics Society.

\section{REFERENCES}

1. Park HW. The implications and significance of the case at Severance hospital. J Korean Med Assoc 2009;52:848-55.

2. Asia Business Daily. Well-dying controversy is raised by Boramae hospital incident [Internet]. Seoul: Asia Business Daily; 2015 [cited 2015 Dec 9]. Available from: http://www.asiae.co.kr/news/view. htm?idxno=2015120909090909325.

3. Mullick A, Martin J, Sallnow L. An introduction to advance care planning in practice. BMJ 2013;347:f6064.

4. Singer PA, Siegler M. Elective use of life-sustaining treatments in internal medicine. Adv Intern Med 1991;36:57-79.

5. Shin HY, Lee JY, Kim JE, Lee S, Youn H, Kim H, et al. Cause-ofdeath statistics in 2016 in the Republic of Korea. J Korean Med Assoc 2018;61:573-84.

6. Jang Y. The elderly and hospice palliative care. Health Welf Policy 
Forum 2015;225:38-47.

7. Hughes DL, Singer PA. Family physicians' attitudes toward advance directives. CMAJ 1992;146:1937-44

8. Masuda Y, Fetters MD, Hattori A, Mogi N, Naito M, Iguchi A, et al. Physicians's reports on the impact of living wills at the end of life in Japan. J Med Ethics 2003;29:248-52.

9. Yee A, Seow YY, Tan SH, Goh C, Qu L, Lee G. What do renal health-care professionals in Singapore think of advance care planning for patients with end-stage renal disease? Nephrology (Carlton) 2011;16:232-8.

10. Sittisombut S, Maxwell C, Love EJ, Sitthi-Amorn C. Physicians' attitudes and practices regarding advanced end-of-life care planning for terminally ill patients at Chiang Mai University Hospital, Thailand. Nurs Health Sci 2009;11:23-8.

11. Asai A, Miura Y, Tanabe N, Kurihara M, Fukuhara S. Advance directives and other medical decisions concerning the end of life in cancer patients in Japan. Eur J Cancer 1998;34:1582-6.

12. Voltz R, Akabayashi A, Reese C, Ohi G, Sass HM. End-of-life decisions and advance directives in palliative care: a cross-cultural survey of patients and health-care professionals. J Pain Symptom
Manage 1998;16:153-62.

13. Coleman AM. Physician attitudes toward advanced directives: a literature review of variables impacting on physicians attitude toward advance directives. Am J Hosp Palliat Care 2013;30:696706.

14. Asai A, Fukuhara S, Inoshita O, Miura Y, Tanabe N, Kurokawa K. Medical decisions concerning the end of life: a discussion with Japanese physicians. J Med Ethics 1997;23:323-7.

15. Yun Y, Lee Y, Nam S, Chae Y, Heo D, Lee S, et al. Public attitudes toward dying with dignity and hospice, palliative care. Korean J Hosp Palliat Care 2004;7:17-28.

16. Silveira MJ, Wiitala W, Piette J. Advance directive completion by elderly Americans: a decade of change. J Am Geriatr Soc 2014;62:706-10.

17. Sulmasy DP, Song KY, Marx ES, Mitchell JM. Strategies to promote the use of advance directives in a residency outpatient practice. J Gen Intern Med 1996;11:657-63.

18. Lee JE, Goo AJ, Cho BL. The current status of end-of-life care in Korea and legislation of well-dying act. 2016;20:65-70. 